\title{
Genotyping more cows increases genetic gain and reduces rate of true inbreeding in a dairy cattle breeding scheme using female reproductive technologies
}

\author{
J. R. Thomasen, ${ }^{1} \oplus$ H. Liu, ${ }^{2 *} \oplus$ and A. C. Sørensen ${ }^{2}$ \\ ${ }^{1}$ VikingGenetics, DK 8960, Assentoft, Denmark \\ ${ }^{2}$ Center for Quantitative Genetics and Genomics, Department of Molecular Biology and Genetics, Faculty of Science and Technology, \\ Aarhus University, DK-8830, Tjele, Denmark
}

\begin{abstract}
Both small dairy cattle populations and dairy cattle populations with a low level of linkage disequilibrium (LD) suffer from low reliability of genomic prediction. In this study, we investigated whether adding more genotyped cows to the reference population influences the rate of genetic gain and rate of inbreeding by affecting the reliability. A standard breeding program with a large reference population and high LD, which mimicked a breeding program for Danish Holstein population, was simulated as a reference. A Danish Jersey population with a small reference population and high LD and a Red Dairy Cattle population with a large reference population and low LD were also simulated. Two additional breeding programs were simulated for Danish Jersey and Red Dairy Cattle populations, where 2,000 additional genotyped cows were included in the population for genomic selection. All 5 simulated breeding programs were initiated by a founder population to generate LD resembling the real LD pattern, followed by a 20-yr conventional progeny-testing scheme with 1,000 or 10,000 genotyped progeny-tested bulls and a 10 -yr genomic selection scheme with or without 2,000 additional genotyped cows. Evaluation criteria were annual monetary genetic gain and rate of true inbreeding. Our results showed that adding more genotyped cows to the reference in dairy cattle populations has the potential to increase genetic gain and reduce the rate of inbreeding, regardless of reference population size and level of LD. However, it is still not possible to reach the same genetic gain as in the simulated Danish Holstein population with either a small reference population or low LD. Our results also showed that in a small reference population with high $\mathrm{LD}$, it is difficult to manage
\end{abstract}

Received May 16, 2019.

Accepted September 23, 2019.

*Corresponding author: Huiming.Liu@mbg.au.dk inbreeding because of lower accuracy compared with the simulated Danish Holstein population and a smaller number of relevant families to select from. Therefore, breeding strategies need to be chosen to match population size and structure. The rate of true inbreeding is always underestimated by pedigree inbreeding and even more in genomic breeding programs, indicating that some forms of genome-wide inbreeding, instead of pedigree-based inbreeding, should be used to monitor inbreeding when genomic selection is implemented.

Key words: inbreeding, genotyped cow, genomic selection, reference population and linkage disequilibrium

\section{INTRODUCTION}

The reliability of genomic prediction mainly depends on the level of linkage disequilibrium (LD) between markers and QTL and the number of reference animals with phenotypes and genotypes in the reference population from which the SNP effects are estimated (Brito et al., 2011; Veroneze et al., 2014). For the application of genomic selection, Danish Holstein (DH) is a more competitive breed in Nordic countries due to a higher level of LD and larger reference population size compared with the other dairy cattle breeds (Brøndum et al., 2011; Lund et al., 2011; Thomasen et al., 2014). In addition, combining Holstein reference populations from different European countries will further increase the reliabilities of genomic predictions, as these populations are closely related (Lund et al., 2011).

Compared with DH, dairy cattle populations with a comparable size of reference population but with a low level of LD, such as Red Dairy Cattle (RDC), are challenged by lower reliability of genomic prediction because more chromosome segments are segregating (Brøndum et al., 2011; Sodeland et al., 2011). Therefore, to reach the same level of reliability of genomic prediction, reference populations of breeds with a low level of LD should be expanded; for example, by add- 
ing genotyped cows with their own phenotypic records to the existing sire reference population (Buch et al., 2012). If the reliability of genomic prediction becomes similar between dairy breeds with high LD and low $\mathrm{LD}$, the rate of inbreeding is expected to be lower in the breeds with low LD because of greater variation in chromosome segments in the population. Although the LD profile is an important factor to determine the reliability of genomic prediction and inbreeding, it has often not been considered in simulation studies. For instance, some simulation studies have used a short period of random mating of 50 to 100 generations to create initial LD (Lund et al., 2009; Cervantes et al., 2016). It is unlikely that simulated genomes with this setting would have a genomic architecture that resembles that of a real population, as they would lack the short span of LD segments, which may lead to potentially misleading conclusions (Daetwyler et al., 2013). In addition, the level of LD at a given distance between loci varies between populations because it is influenced by the population's evolutionary history and effective population size, which reflects inbreeding levels of the population (Slatkin 2008; Chitneedi et al., 2017). Thus, the LD profile needs to be taken into account in a simulation study to make reasonable breeding programs.

Compared with DH and RDC, small dairy cattle populations with a high level of LD, such as Danish Jersey (DJ), are often restricted by small reference populations (Thomasen et al., 2014). These populations, therefore, have low reliabilities of genomic prediction. One option to increase reference population size may be to join 2 or more populations from different breeds into a common reference population, without the need to add extra costs for genotyping and phenotyping. However, the results have shown that little or no benefit is gained when combining breeds such as Holstein and Jersey because of the distant relationships between the breeds (Lund et al., 2014). Besides a joint reference population across breeds, 2 solutions, as shown in Thomasen et al. (2014), can be considered to expand the reference population: (1) increasing the annual number of progeny-tested bulls included in the reference population, and (2) adding genotyped females with their own phenotypic records to the existing sire reference population. Although both can increase genetic gain and reduce rate of inbreeding due to higher reliabilities of genomic predictions, the latter is expected to reduce rate of inbreeding more than the former due to a more-efficient within-family selection and less between-family selection (Daetwyler et al., 2007; Thomasen et al., 2014). In addition, adding progeny-tested bulls to the reference population may not increase the genetic gain because of the risk of reducing selection intensity. Therefore, adding genotyped females to the reference population should be a more appropriate strategy to make a small dairy cattle population competitive.

In this study, we hypothesize that using more genotyped females in the reference population will increase the reliabilities of genomic prediction and, therefore, enhance the rates of genetic gain while reducing true and pedigree inbreeding. We simulated populations having a reference population size and LD level that resemble that of real $\mathrm{DH}, \mathrm{RDC}$, and $\mathrm{DJ}$ populations. The DH population was used as the reference, as the other 2 breeds are challenged in different ways. The level of inbreeding was evaluated based on genome-wide homozygosities due to identity-by-descent (IBD; true inbreeding) and was also estimated with pedigree information (pedigree inbreeding).

\section{MATERIALS AND METHODS}

\section{Experimental Design}

To test the hypotheses, we simulated breeding programs consisting of 3 stages. In the first stage, we modeled 3,000 generations of a founder population to generate the LD profile and genomic architecture. In the second stage, we simulated a conventional progenytesting scheme for $20 \mathrm{yr}$, building a reference population with 1,000 (small reference population) or 10,000 (large reference population) genotyped progeny-tested bulls. In the third stage, we simulated a genomic selection scheme for yr 21 to 30 . In the standard breeding programs, 2,000 genotyped cows were added yearly to the reference population. The 3 scenarios with different combinations of LD level and reference population size were as follows: (1) reference (Ref; high LD and a large reference population), small (Sm; high LD and a small reference population), and diverse (Div; low LD and a large reference population). These 3 scenarios represented DH, DJ, and RDC, respectively. A small population was only simulated under high LD, as a small population size will augment LD by increasing the effect of genetic drift (Slatkin, 2008). To evaluate the effect of increasing the reliabilities of genomic predictions, the number of genotyped cows was doubled yearly, designated as the "Xcows" scenarios. Thus, 2 more breeding scenarios, Xcows-Sm and Xcows-Div, were added. The details of the 5 breeding programs are given in Table 1.

\section{LD Profile}

In practice, DH and DJ populations have similar LD profiles and both have a higher level of LD than the 
Table 1. Details of the simulation of the 5 breeding programs that differ in level of linkage disequilibrium (LD), size of reference population, the use of extra genotyping cows, and the breed mimicked

\begin{tabular}{lllll}
\hline $\begin{array}{l}\text { Breeding } \\
\text { program }\end{array}$ & $\begin{array}{l}\text { LD } \\
\text { level }\end{array}$ & $\begin{array}{l}\text { Reference } \\
\text { population size }\end{array}$ & $\begin{array}{l}\text { Extra } \\
\text { cows }\end{array}$ & Breed \\
\hline Small & High & Small & No & Danish Jersey \\
Xcows-Small & High & Small & Yes & Danish Jersey \\
Reference & High & Large & No & Danish Holstein \\
Xcows-Diverse & Low & Large & Yes & Red dairy cattle \\
Diverse & Low & Large & No & Red dairy cattle \\
\hline
\end{tabular}

RDC population (Figure 1). Therefore, 2 simulation groups were established: a high-LD group, mimicking the real LD profile in $\mathrm{DH}$ and DJ populations, and a low-LD group, mimicking the RDC population. The squared correlation coefficient, $\mathrm{r}^{2}$, was used as a measure of LD and was calculated, as described in Thomasen et al. (2013), as the squared correlations between markers grouped into bins by lengths of $7 \mathrm{~kb}$. In this paper, the genome-wide LD profile was only published for DJ, including 1,730 animals with 39,824 markers. For DH, 9,589 animals with 38,741 markers were included in the calculations, and for RDC, 6,581 animals with 43,621 markers were used. Calculations of the LD pattern for DH and RDC are as described in Thomasen et al. (2013).

a

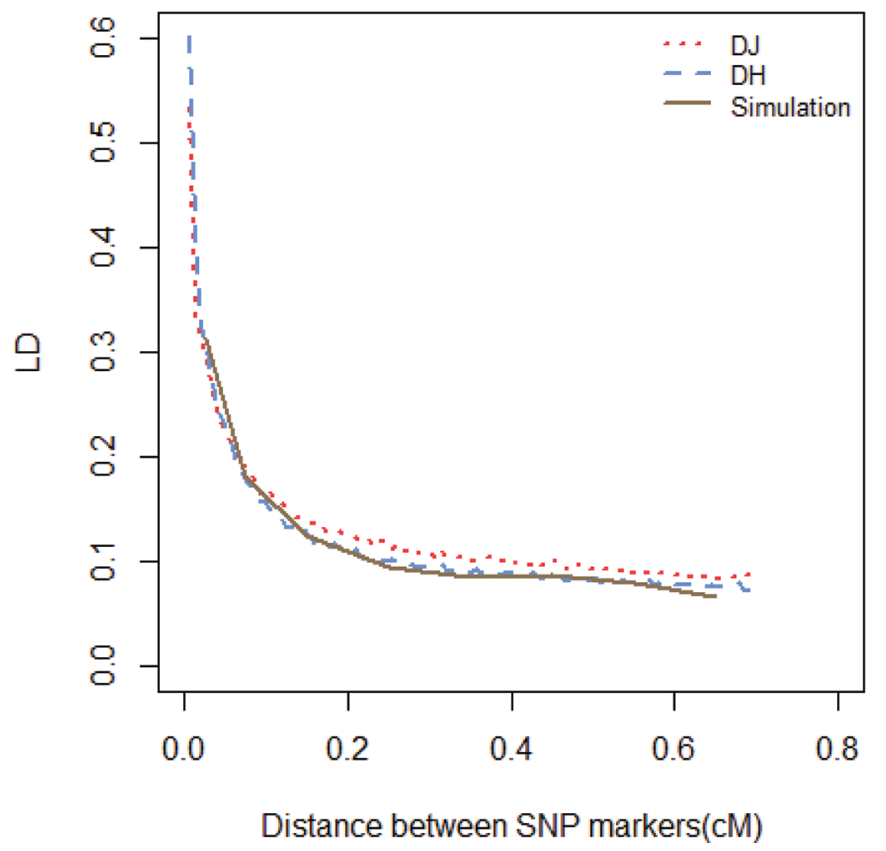

\section{Simulations}

Founder Population and Genetic Architecture. The populations with high and low LD were simulated based on forward-in-time process with 3,000 generations and with equal size of each sex. The simulated genome consisted of thirty 1-M-long chromosomes, on which 100 QTL and 10,000 biallelic SNP marker loci were randomly distributed, resulting in 3,000 QTL and 300,000 SNP marker loci across the genome. The offspring inherited alleles at these loci from their parents, following Mendelian rules and allowing for mutation and recombinations. A recurrent mutation rate of 2.5 $\times 10^{-5}$ per locus per meiosis was performed to establish mutation-drift equilibrium in founder generations. The

b

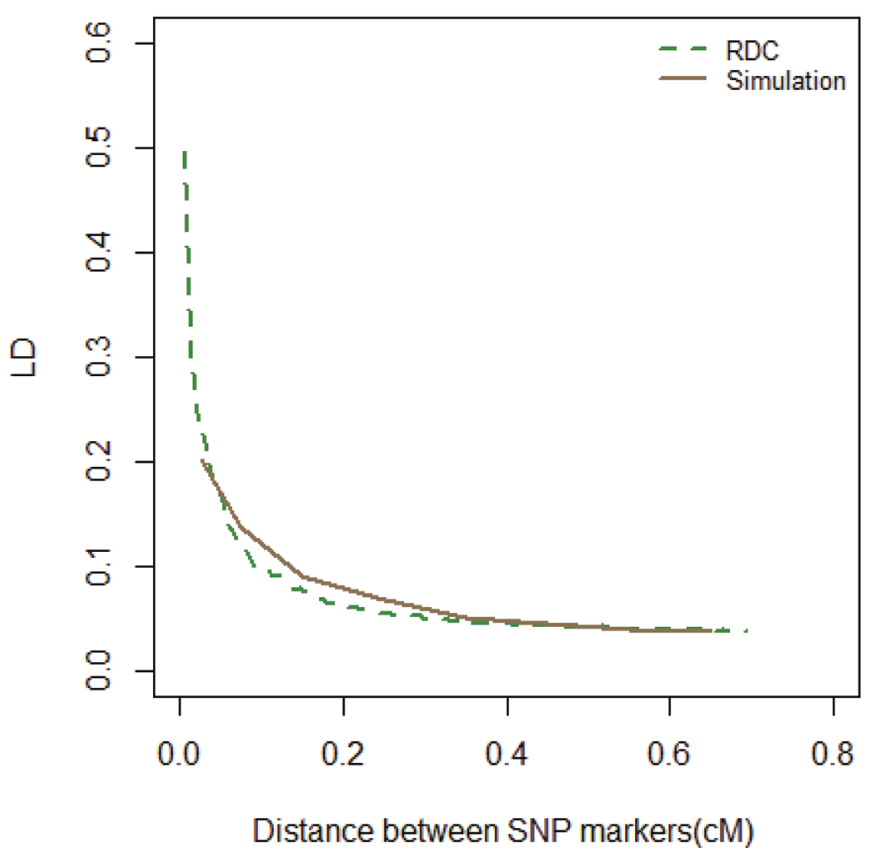

Figure 1. Linkage disequilibrium (LD) calculated as average $\mathrm{r}^{2}$ (squared correlation coefficient) for different marker distances (cM) between each pair of markers in (a) Danish Jersey (DJ) and Danish Holstein (DH) populations; the gray solid line shows the $\mathrm{r}^{2}$ in the last generation of simulated founder population with higher LD; and (b) Red Dairy Cattle (RDC) population; the gray solid line shows the $\mathrm{r}^{2}$ in the last generation of simulated founder population with lower LD. 
Table 2. Parameters used in modeling the different population structures and breeding programs

\begin{tabular}{|c|c|c|c|c|c|}
\hline Population structure & $\begin{array}{l}\text { Program 1: } \\
\text { Small }\end{array}$ & $\begin{array}{l}\text { Program 2: } \\
\text { Xcows-Small }\end{array}$ & $\begin{array}{l}\text { Program 3: } \\
\text { Reference }\end{array}$ & $\begin{array}{c}\text { Program 4: } \\
\text { Xcows-Diverse }\end{array}$ & $\begin{array}{l}\text { Program 5: } \\
\text { Diverse }\end{array}$ \\
\hline \multicolumn{6}{|l|}{ Step 1: Founder populations } \\
\hline Population size after a bottleneck & 50 & 50 & 50 & 600 & 600 \\
\hline No. of progeny-tested young bulls & 50 & 50 & 500 & 500 & 500 \\
\hline No. of proven bulls selected per year & 5 & 5 & 20 & 20 & 20 \\
\hline \multicolumn{6}{|l|}{ Step 3: Genomic breeding scheme } \\
\hline No. of proven bulls in reference yr 30 & 1,600 & 1,600 & 10,600 & 10,600 & 10,600 \\
\hline No. of cows in reference yr 30 & 16,000 & 32,000 & 16,000 & 16,000 & 32,000 \\
\hline
\end{tabular}

recombinations per chromosome were sampled from a Poisson distribution with a mean equal to the length of the chromosome (in Morgans) and were randomly placed along the chromosome assuming a uniform distribution.

To obtain a similar LD profile as in the real data, several alternatives were run to calibrate LD in the simulations by varying the expansion and bottleneck of the founder population. After testing the alternatives, the following populations were the best match and used as the founder population. For the population with high LD, the first 100 generations were generated with a gradual decrease in population size from 1,200 to 200, followed by 2,800 generations with a linear expansion of the population to 3,000 . This was done to match the peak of LD between adjacent loci in the real data. In the last 100 generations, a bottleneck was simulated, where the population size decreased linearly from 3,000 to 50 . This was done based on the estimation by VillaAngulo et al. (2009) and to match the average level of LD between simulated and real data. During these 3,000 generations, the numbers of males and females remained the same. For the population with low LD, the only difference from the high-LD population was that in the last 100 generations, the population size decreased linearly from 3,000 to 600 instead of 50 to obtain low LD.

After 3,000 generations, we calculated LD between each pair of markers that were still segregating in the population. A comparison of the LD profile based on the simulation data with that obtained based on the real marker data is given in Figure 1. In generation 3,000 , among all the segregating loci, a set of 40,000 SNP loci across the genome were randomly chosen as SNP markers and another set of 1,000 loci were randomly chosen as QTL, each having a small but noninfinitesimal effect. The minor allele frequencies ranged from 0.0017 to 0.5 for markers and from 0.02 to 0.5 for QTL. Generation 3,000 was used as the base population for the subsequent steps.
Conventional Progeny-Testing Scheme. A detailed description of the parameters used in the 2 conventional breeding scenarios (small and large reference populations) is given in Table 2. For the proven-bull schemes, only daughter proven bulls were used as sires of sons. In the scenario with a small reference population, each year, 50 young bulls were progeny-tested and 5 bulls were selected as proven bulls ( 5 to 6 yr old). In the scenario with a large reference population, each year, 500 bulls were progeny-tested and 20 bulls were selected as proven bulls. All progeny-tested bulls were genotyped and included in the sire reference population for the genomic selection scheme.

Genomic Selection Scheme. The genomic breeding schemes were similar for all strategies to study how inbreeding is influenced by different population structures and the reliability of genomic EBV of the breeding goal (GEBV) without any influence by the breeding plan. In all breeding programs, the simulated cow population consisted of 10,000 cows with records from 100 herds, reflecting the part of the cow population where the bull calves are recruited. The genomic breeding scheme follows the selection steps described in Thomasen et al. (2016), with use of reproductive technologies on the highest-ranking females according to their total merit index as described in the next paragraph. In the present scheme (Table 2), the 200 highest-ranking females were used as donors to produce 10 progeny each at 23 mo of age. A total of 2,000 bull calves were genotyped yearly for selection of 100 bulls for semen production and 2,000 cows were genotyped yearly. Selections of bull calves and females for genotyping were based on ranking their total merit index from pedigree. Numbers of bulls and cows with phenotypes included in the reference population in the last year of the simulation are listed in Table 2.

Breeding Goal and Breeding Values. All animals were selected according to the breeding goal, which included 2 traits: a trait representing milk production traits $\left(h^{2}=0.30\right)$ and a trait representing functional 
traits $\left(h^{2}=0.04\right)$. An unfavorable genetic correlation of -0.30 between the 2 traits was assumed. The effects of the QTL on the 2 traits were sampled from a bivariate normal distribution with mean 0 and then scaled to a variance equal to 1 and resulted in the specified genetic parameters for the 2 traits. The residuals were assumed uncorrelated. The economic values were set to $€ 83$ and $€ 82$ for the 2 traits (milk production and functionality), respectively, per additive genetic standard deviation. In both conventional schemes, the progeny-tested bulls obtained 150 daughters for the production trait and 135 daughters for the functional trait. A more detailed description of the traits and sampling of breeding values and phenotypes can be found in Buch et al. (2012) and Thomasen et al. (2014). The DMU software package was used to predict breeding values using single-step genomic BLUP (Madsen and Jensen, 2013), following the procedures described in Thomasen et al. (2014).

\section{Evaluation Criteria}

The stochastic simulation tool ADAM (Pedersen et al., 2009) was used to simulate the different breeding programs. Each of the 5 strategies was replicated 20 times. Analyses of annual monetary genetic gain $(\boldsymbol{\Delta} \boldsymbol{G})$ was performed using the same breeding program as in Thomasen et al. (2014). The rate of inbreeding per generation $(\boldsymbol{\Delta} \boldsymbol{F})$ was evaluated in yr 11 to 20 in the proven-bull schemes and in yr 26 to 30 in the genomic schemes.

To evaluate true inbreeding, we simulated IBD markers that were evenly distributed across the genome, as described in Liu et al. (2017). These IBD markers were not involved in selection, but were assigned unique alleles to each base animal. They were used to trace each base animal's contribution to their descendant generations and infer IBD status relative to the base population. Thus, within each locus of a descendant, each IBD marker allele could be traced directly back to the base animal from which it was derived. Any homozygous locus at IBD markers was an inbred locus. Inbreeding at each IBD marker was defined as the probability that 2 alleles at that locus from a randomly selected animal in the population are IBD.

For each replicate, $\Delta G$ was calculated as the regression coefficient of mean true breeding value on birth year of animal. In this study, $\Delta G$ was presented as the mean of replicates for each scenario. Rates of inbreeding were presented per generation and estimated from the pedigree $\left(\boldsymbol{\Delta} \boldsymbol{F}_{\text {ped }}\right)$ and from IBD markers $\left(\boldsymbol{\Delta} \boldsymbol{F}_{\text {true }}\right)$. The inbreeding rate per generation was calculated for each replicate as 1 minus the exponential of the regression coefficient of the natural logarithm of mean inbreeding on the average generation equivalent for each year (Liu et al., 2017). The presented inbreeding was then obtained by averaging over replicates. Differences in $\Delta G, \Delta F_{\text {ped }}$, and $\Delta F_{\text {true }}$ between scenarios were compared with the least significant difference (LSD) using a confidence level of $95 \%$.

The reliabilities of GEBV were calculated as the squared correlation between the EBV and true breeding values of the available candidates in a specific selection group. The reliabilities were calculated as averages across replicates within each selection group and birth year.

\section{RESULTS}

\section{Genetic Gain and Genomic Reliability}

In the genomic selection stage, the Ref breeding program provided the significantly highest $(P<0.05) \Delta G$ of $€ 42.73$ (Table 3 ). The average reliability of GEBV was highest $(0.49 ; P<0.05)$ for this breeding program. Lowering the LD level reduced the $\Delta G$ to $€ 38.84$ for the Div scenario. Doubling the number of genotyped cows in the reference population (Xcows-Div) marginally increased the $\Delta G$ by $0.34(0.9 \%)$ to $€ 39.18$. However, this increase in $\Delta G$ was not significantly different and the average reliability was not significantly higher. The Sm breeding program showed the significant lowest $(P<0.05) \Delta G(€ 35.56)$ for all the genomic schemes, which was $17 \%$ lower than that of Ref. Doubling the number of cows (Xcows-Sm) increased the genetic gain significantly $(P<0.05)$ by $6 \%$ to $€ 37.64$, which was at the same level as Div. None of the Xcows strategies reached the same level of $\Delta G$ as Ref. The $\Delta G$ for the conventional selection stage was approximately 43 to $49 \%$ lower than that for the genomic selection stage.

Figure 2 shows the development in reliabilities of GEBV for the last $6 \mathrm{yr}$ of the genomic selection stage. For Ref, there was a slight decrease in reliability. For Div, the reliabilities were constant over time. For Sm, there was an increase over time of 0.05 percentage points and the same reliability was reached as for Div. From yr 28 , there was a positive effect on the reliability of adding more genotyped cows to the reference population. Generation interval was 2.44 to $2.52 \mathrm{yr}$ at the genomic selection stage and 4.66 to $4.69 \mathrm{yr}$ at the conventional selection stage.

\section{Rate of Inbreeding}

At the genomic selection stage, the breeding programs with a large reference population had $\Delta F_{\text {true }}$ up to $55.6 \%$ less than those with a small reference population. When the number of genotyped cows was doubled, average $\Delta F_{\text {true }}$ was decreased by 10.1 to $14.8 \%$, regardless of the 
size of the reference population, although the decrease was not statistically significant. The standard deviation of $\Delta F_{\text {true }}$ decreased when the number of genotyped cows was doubled. With a large reference population and low $\mathrm{LD}$, doubling the number of genotyped cows resulted in similar $\Delta F_{\text {true }}$ to the Ref breeding program. Compared with conventional selection stage with progeny testing, genomic selection presented lower $\Delta F_{\text {true }}$ in general.

The difference in $\Delta F_{\text {true }}$ between large and small reference populations was not captured by $\Delta F_{p e d}$, although the ranking of the 5 breeding programs was the same as for $\Delta F_{\text {true }}$. At the conventional stage, there was no significant difference between $\Delta F_{\text {true }}$ and $\Delta F_{\text {ped }}$. However, $\Delta F_{\text {ped }}$ always underestimated $\Delta F_{\text {true }}$ at the genomic selection stage.

\section{Inbreeding Over Time}

Although breeding programs with a large reference population resulted in lower inbreeding over time compared with a small reference population, the pattern of inbreeding development was similar across the 5 breeding programs (Figure 3), where $F_{\text {true }}$ and $F_{\text {ped }}$ started to diverge from generation 22 , and $F_{\text {true }}$ became increasingly higher compared with $F_{\text {ped }}$ over time with genomic selection.

\section{DISCUSSION}

Our main hypothesis was confirmed: increasing the number of genotyped females included in the reference population generally increased $\Delta G$ and reduced $\Delta F_{\text {true }}$. The increase in $\Delta G$ by doubling the number of genotyped females was more pronounced in the Sm breeding program (6\%) than in Div (1\%). However, it was not possible to reach the same level of genetic gain as in Ref, even when doubling the number of cows added to the reference in either $\mathrm{Sm}$ or Div. The $\Delta F_{\text {true }}$ was reduced by 10 to $15 \%$ when doubling the number of genotyped females under Sm and Div. The $\Delta F_{\text {true }}$ in
Xcows-Div was controlled at almost the same level as in Ref, whereas $\Delta F$ in Xcows-Sm was approximately twice as large as in Xcows-Div and Ref. This suggested that it is difficult to manage inbreeding under Xcows-Sm because of the lower accuracy and a smaller number of relevant families to select from.

\section{Genetic Gain}

We found the highest increase in $\Delta G$ by adding more genotyped cows to the reference in $\mathrm{Sm}$. In addition, the reliability of GEBV was increased over time. This is because the sire reference population is relatively small with only 1,000 bulls combined with the population with high LD structure, which provided each genotyped cow with a relatively high marginal value. However, the genetic gain was still $11.9 \%$ below that for Ref, showing that it is very difficult for a small population to obtain the same genetic progress as a large population that has a large reference population of progeny-tested bulls. Over time, the bull reference population becomes less important because of changes in allele frequencies, as evidenced by the decrease in average reliability in the Ref. scenario. Our simulation results indicated that small and large populations become more alike in accuracy and therefore should also become more alike in $\Delta G$. In contrast to the Div population, the increase in $\Delta G$ by adding more genotyped cows to the reference population in general was only marginal. This may be because the effect of having more females to update and enlarge the reference population in general had less effect when a large number of progeny-tested bulls have already been included in the reference population. Furthermore, the low level of LD (Figure 1) made the marginal value of the individual genotyped cows less. It is more difficult to increase genetic gain when the population has low LD. This means that diverse populations need more investments in genotyping of phenotyped cows to compete with large, more inbred populations. Alternative and less costly methods would

Table 3. Number of replicates $(N r e p)$ and rates of pedigree inbreeding $\left(\Delta F_{\text {ped }}\right)$, true inbreeding $\left(\Delta F_{\text {true }}\right)$, and genetic gain $(\Delta G)$ over the first 20 yr (conventional selection stage) and over the last $10 \mathrm{yr}$ (genomic selection stage) for different breeding schemes (SD in parentheses)

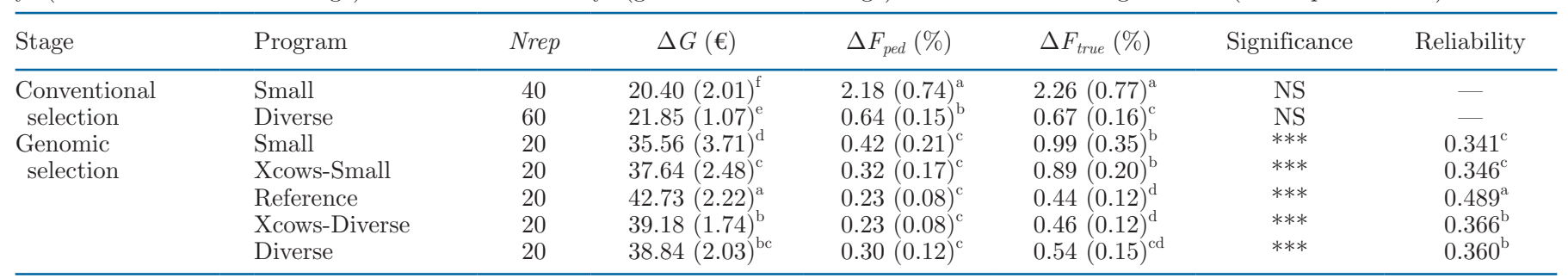

\footnotetext{
${ }^{\mathrm{a}-\mathrm{f}}$ Values within a column with different superscripts differ $(P<0.05)$

*** $P \leq 0.001$ : significance of difference between $\Delta F_{\text {ped }}$ and $\Delta F_{\text {true }}$ for the breeding program.
} 
be to join reference data from different breeds, which would require similarities in LD structures and good genetic links (Lund et al., 2016).

\section{Rate of True Inbreeding and Pedigree Inbreeding}

The $\Delta F_{\text {true }}$ in Sm was significantly higher than that in Div because of 2 possible mechanisms. The first is that with $\mathrm{Sm}$, there is a higher chance of co-selection of families because of the lower reliability of Mendelian sampling terms compared with Div. The second is that with high LD, the population contains more lowdiversity regions and is more affected by the hitchhiking effect due to linkage drag compared with low LD. Hence, there are fewer opportunities to obtain an optimal combination of haplotypes. Both mechanisms can result in less within-family selection and more acrossfamily selection. Therefore, less diversity is maintained in the Sm scenario and $\Delta F_{\text {true }}$ is increased. In both $\mathrm{Sm}$ and Div scenarios, with more females genotyped, there is higher reliability of the Mendelian sampling term, which also results in more within-family selection and lower $\Delta F_{\text {true }}$.

Another important finding regarding inbreeding was that there was almost no difference between $\Delta F_{p e d}$ and $\Delta F_{\text {true }}$ at the conventional selection stage for all breeding programs, whereas there was a large and significant difference between $\Delta F_{p e d}$ and $F_{\text {true }}$ at the genomic selection stage. In the conventional breeding scheme, BLUP was performed, which depends on the numerator relationship derived from the pedigree. Pedigree inbreeding was also computed based on the numerator relationship, which can effectively capture unequal contributions per animal for a neutral locus that is unlinked to variants with an effect and development of numerator relationship due to BLUP selection. Sonesson et al.

\section{Mean reliability of GEBV}

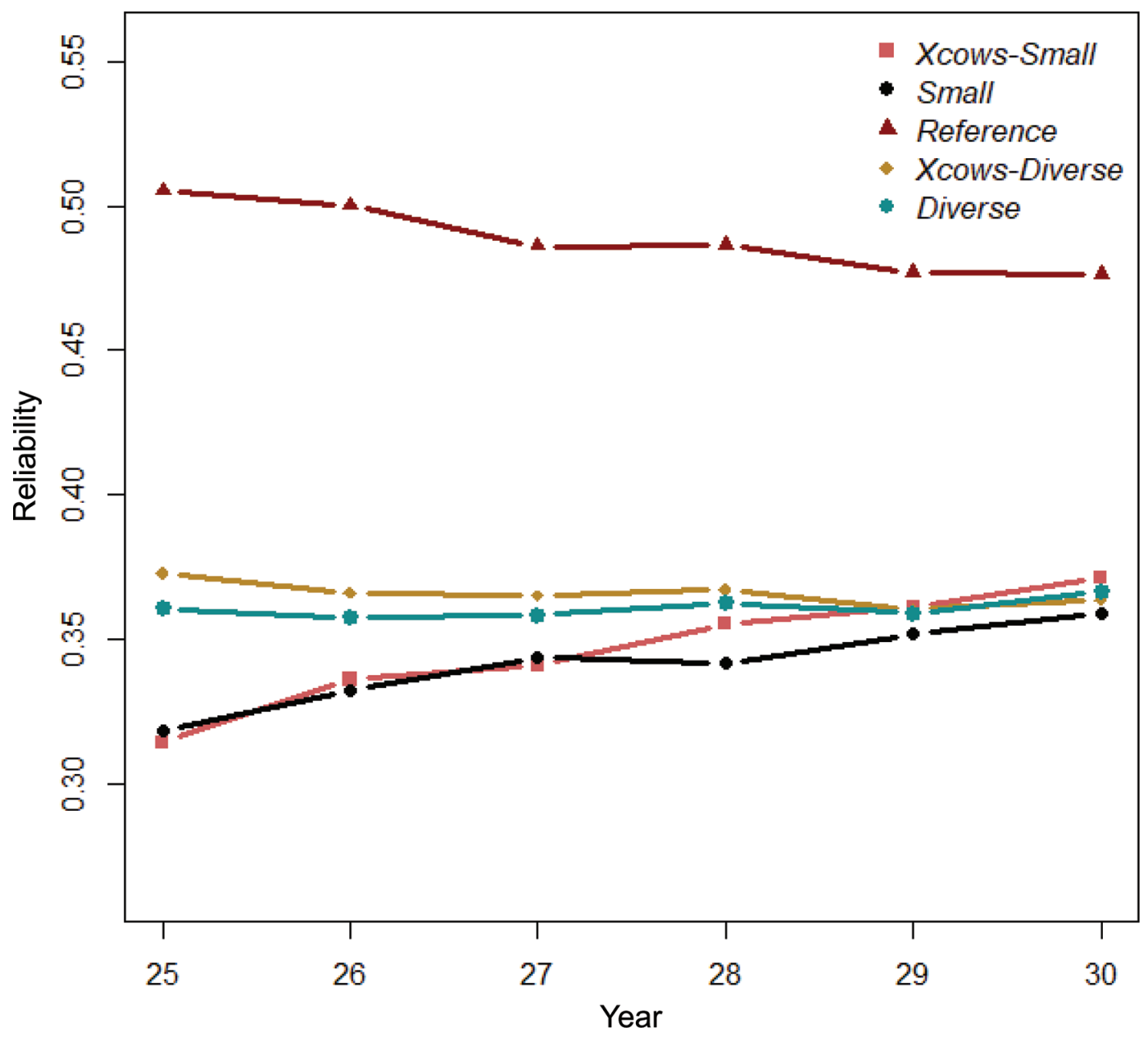

Figure 2. Mean of reliabilities of genomic EBV (GEBV) for the 5 breeding programs (defined in Table 1). 
(2012) and Liu et al. (2014) found that when BLUP was performed, the difference between $F_{p e d}$ and $F_{\text {true }}$ was relatively small compared with genomic selection. Therefore, under BLUP without genomic information, pedigree inbreeding is a relatively good estimate of true inbreeding.

In the genomic breeding scheme, however, estimation of GEBV depends on the identity-by-state relationship obtained from the markers accumulated over time. True inbreeding $\left(F_{\text {true }}\right)$ can capture the relationship between the IBD markers defined in the base population used for reference and capture the unequal contributions of alleles per marker locus. It is an empirical value that is not restricted by neutrality or position of the locus. Pedigree inbreeding cannot predict the Mendelian segregation of each marker locus and therefore would fail to capture the relationship between the whole-genome IBD loci and underestimate the relationships in the region due to nonrandom within-family selection. There- fore, some form of genome-wide inbreeding, instead of pedigree-based inbreeding, needs to be used to monitor inbreeding when genomic selection is implemented.

Our results confirmed that $\Delta F_{\text {ped }}$ underestimates $\Delta F_{\text {true }}$ under both Sm and Div in the genomic breeding scheme. With Sm, $\Delta F_{\text {true }}$ was substantially underestimated by 58 to $64 \%$, and with Div, $\Delta F_{\text {true }}$ was underestimated by 44 to $50 \%$. This may be due to a larger hitchhiking effect by which fewer neutral loci exist in the population. This also suggests that management of inbreeding is important under $S m$ to prevent expression of detrimental effects associated with inbreeding.

\section{Expectations of Inbreeding in Genomic Breeding Schemes}

Whether genomic selection will increase or decrease $\Delta F$ compared with conventional breeding schemes has been a topic of debate in the literature (Schaeffer,

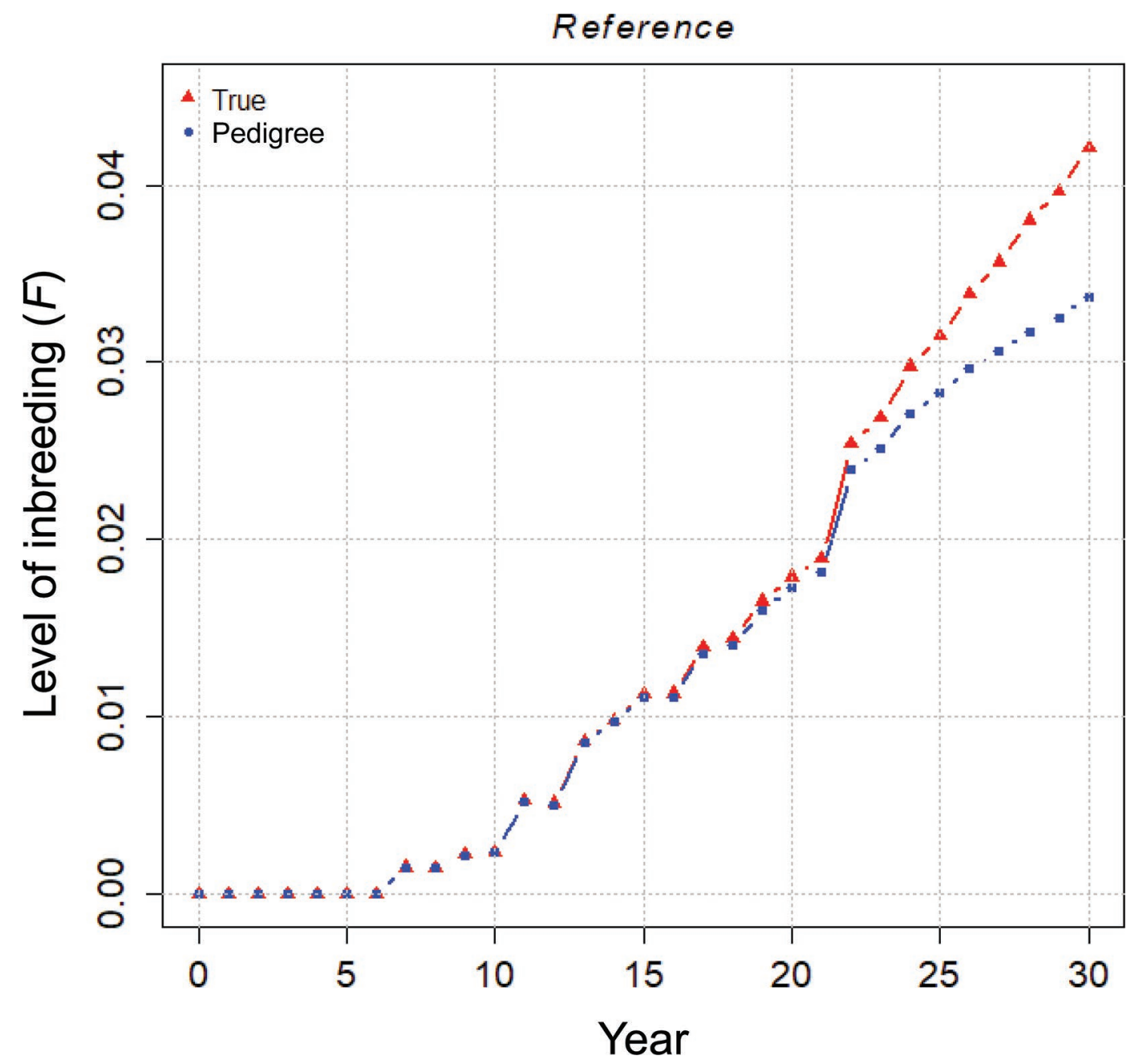

Figure 3. The trend of pedigree $\left(F_{p e d}\right)$ and true inbreeding $\left(F_{\text {true }}\right)$ for the reference (Ref) breeding program. 
2006; Daetwyler et al., 2007; de Roos et al., 2011). A recent study on Dutch Holstein-Friesian data showed that rate of inbreeding per generation increased after the introduction of genomic selection. The result was consistent for different inbreeding measures; that is, pedigree-based (1.8\%), runs of homozygosity-based (2.1\%), and marker-based (2.8\%) calculated from 2010 and onward (Doekes et al., 2018). These findings were notably higher than those found in our study, where the Sm population generated the largest $\Delta F_{\text {true }}$ of $0.99 \%$.

The obtained $\Delta F$ was highly affected by the applied breeding program, including the number of bulls used per generation. Using genomic information as a preselection tool with no change in breeding scheme compared with the conventional progeny-testing scheme, a lower $\Delta F$ was expected because of the higher accuracy of young genomic bulls. The $\Delta F$ is expected to increase in a full genomic scheme due to the lower generation interval and a lower selection accuracy because no progeny-tested bulls with high accuracy are used. Selecting breeding animals with a lower selection accuracy results in less within-family selection and more across-family selection; that is, favoring specific families, which results in higher inbreeding (Daetwyler et al., 2013). To obtain a lower $\Delta F$ per generation, more bulls per generation need to be selected. In the present study, 94 sires of sons were selected per generation for Ref and Div and 24 for Sm. In the genomic scheme, 252 sires of sons were selected per generation because all genomic-selected bulls were used as sires of sons. In a study by Thomasen et al. (2016), a variety of genomic breeding schemes were investigated in pseudo genomic setup. The scheme in Thomasen et al. (2016), matching the genomic scheme Ref, provided a $\Delta G$ of $€ 41.4$ and $\Delta F$ of $0.24 \%$, which is close to the values found in this study. Reducing the number of bulls from 100 to 50 increased $\Delta G$ to $€ 44.2(+7 \%)$ but increased $\Delta F$ relatively by a much greater degree, to $0.44 \%(+83 \%)$. Therefore, lower selection accuracy in genomic schemes can be compensated for by selecting more bulls. The marginal loss in $\Delta G$ is only minor, whereas the reduction in $\Delta F$ is major.

\section{CONCLUSIONS}

Adding more genotyped cows to reference dairy cattle populations has the potential to increase genetic gain and reduce inbreeding, regardless of reference population size and level of LD. However, it is still not possible to reach the same genetic gain as simulated in the $\mathrm{DH}$ population with either a small reference population or low LD. Our results suggested that with a small reference population and high LD, it is difficult to manage inbreeding due to lower accuracy and a smaller number of relevant families to select from. Therefore, breeding strategies need to be chosen that match population size and structure. The rate of true inbreeding is always underestimated by pedigree inbreeding, suggesting that some forms of genome-wide inbreeding, instead of pedigree-based inbreeding, should be used to monitor inbreeding when genomic selection is implemented.

\section{ACKNOWLEDGMENTS}

This project was funded by The Danish Council for Strategic Research (Aarhus, Denmark), Center for Genomic Selection in Animals and Plants (GenSAP; grant no. 12-132452; Aarhus, Denmark) and Green Development Program (GUDP; grant nos. 34009-13-0607 and 34009-15-0951; Aarhus, Denmark).

\section{REFERENCES}

Brito, F. V., J. Neto, M. Sargolzaei, J. Cobuci, and F. Schenkel. 2011. Accuracy of genomic selection in simulated populations mimicking the extent of linkage disequilibrium in beef cattle. BMC Genet. 12:80. https://doi.org/10.1186/1471-2156-12-80.

Brøndum, R. F., E. Rius-Vilarrasa, I. Strandén, G. Su, B. Guldbrandtsen, W. F. Fikse, and M. S. Lund. 2011. Reliabilities of genomic prediction using combined reference data of the Nordic Red dairy cattle populations. J. Dairy Sci. 94:4700-4707. https://doi.org/10 .3168/jds.2010-3765.

Buch, L. H., M. Kargo, P. Berg, J. Lassen, and A. C. Sørensen. 2012 The value of cows in reference populations for genomic selection of new functional traits. Animal 6:880-886. https://doi.org/10.1017/ S1751731111002205.

Cervantes, I., J. P. Gutiérrez, and T. H. E. Meuwissen. 2016. Response to selection while maximizing genetic variance in small populations. Genet. Sel. Evol. 48:69. https://doi.org/10.1186/s12711-016 $-0248-3$.

Chitneedi, P. K., J. J. Arranz, A. Suarez-Vega, E. García-Gámez, and B. Gutiérrez-Gil. 2017. Estimations of linkage disequilibrium, effective population size and $\mathrm{ROH}$-based inbreeding coefficients in Spanish Churra sheep using imputed high-density SNP genotypes. Anim. Genet. 48:436-446. https://doi.org/10.1111/age.12564.

Daetwyler, H. D., M. P. L. Calus, R. Pong-Wong, G. de los Campos, and J. M. Hickey. 2013. Genomic prediction in animals and plants: simulation of data, validation, reporting and bench marking. Genetics 193:347-365. https://doi.org/10.1534/genetics.112.147983.

Daetwyler, H. D., B. Villanueva, P. Bijma, and J. A. Woolliams. 2007. Inbreeding in genome-wide selection. J. Anim. Breed. Genet. 124:369-376. https://doi.org/10.1111/j.1439-0388.2007.00693.x.

de Roos, A. P. W., C. Schrooten, R. F. Veerkamp, and J. A. M. van Arendonk. 2011. Effects of genomic selection on genetic improvement, inbreeding, and merit of young versus proven bulls. J. Dairy Sci. 94:1559-1567. https://doi.org/10.3168/jds.2010-3354.

Doekes, H. P., R. F. Veerkamp, P. Bijma, S. J. Hiemstra, and J. J. Windig. 2018. Trends in genome-wide and region-specific genetic diversity in the Dutch-Flemish Holstein-Friesian breeding program from 1986 to 2015. Genet. Sel. Evol. 50:15. https://doi.org/10 .1186/s12711-018-0385-y.

Liu, H., M. Henryon, and A. C. Sorensen. 2017. Mating strategies with genomic information reduce rates of inbreeding in animal breeding schemes without compromising genetic gain. Animal 11:547-555. https://doi.org/10.1017/S1751731116001786.

Liu, H., A. C. Sørensen, T. H. E. Meuwissen, and P. Berg. 2014. Allele frequency changes due to hitch-hiking in genomic selection programs. Genet. Sel. Evol. 46:8. https://doi.org/10.1186/1297 $-9686-46-8$. 
Lund, M. S., A. de Roos, A. de Vries, T. Druet, V. Ducrocq, S. Fritz, F. Guillaume, B. Guldbrandtsen, Z. Liu, R. Reents, C. Schrooten, F. Seefried, and G. Su. 2011. A common reference population from four European Holstein populations increases reliability of genomic predictions. Genet. Sel. Evol. 43:43. https://doi.org/10.1186/1297 $-9686-43-43$

Lund, M. S., G. Sahana, D. J. de Koning, G. Su, and O. Carlborg. 2009. Comparison of analyses of the QTLMAS XII common dataset. I: Genomic selection. BMC Proc. 3(Suppl. 1):S1. https://doi .org/10.1186/1753-6561-3-S1-S1.

Lund, M. S., G. Su, L. Janss, B. Guldbrandtsen, and R. F. Brøndum. 2014. Genomic evaluation of cattle in a multi-breed context. Livest. Sci. 166:101-110. https://doi.org/10.1016/j.livsci.2014.05.008.

Lund, M. S., I. van den Berg, P. Ma, R. F. Brøndum, and G. Su. 2016. Review: How to improve genomic predictions in small dairy cattle populations. Animal 10:1042-1049. https://doi.org/10.1017/ S1751731115003031.

Madsen, P., and J. Jensen. 2013. A User's Guide to DMU. Version 6 , release 5.2. Aarhus University, Foulum, Denmark.

Pedersen, L. D., A. C. Sørensen, M. Henryon, S. Ansari-Mahyari, and P. Berg. 2009. ADAM: A computer program to simulate selective breeding schemes for animals. Livest. Sci. 121:343-344. https://doi .org/10.1016/j.livsci.2008.06.028.

Schaeffer, L. R. 2006. Strategy for applying genome-wide selection in dairy cattle. J. Anim. Breed. Genet. 123:218-223. https://doi.org/ 10.1111/j.1439-0388.2006.00595.x.

Slatkin, M. 2008. Linkage disequilibrium-Understanding the evolutionary past and mapping the medical future. Nat. Rev. Genet. 9:477-485. https://doi.org/10.1038/nrg2361.

Sodeland, M., M. Kent, B. J. Hayes, H. Grove, and S. Lien. 2011. Recent and historical recombination in the admixed Norwegian Red cattle breed. BMC Genomics 12:33. https://doi.org/10.1186/ 1471-2164-12-33.
Sonesson, A. K., J. A. Woolliams, and T. H. E. Meuwissen. 2012. Genomic selection requires genomic control of inbreeding. Genet. Sel. Evol. 44:27. https://doi.org/10.1186/1297-9686-44-27.

Thomasen, J. R., A. C. Sørensen, M. S. Lund, and B. Guldbrandtsen. 2014. Adding cows to the reference population makes a small dairy population competitive. J. Dairy Sci. 97:5822-5832. https://doi .org/10.3168/jds.2014-7906.

Thomasen, J. R., A. C. Sørensen, G. Su, P. Madsen, M. S. Lund, and B. Guldbrandtsen. 2013. The admixed population structure in Danish Jersey dairy cattle challenges accurate genomic predictions. J. Anim. Sci. 91:3105-3112. https://doi.org/10.2527/jas .2012-5490.

Thomasen, J. R., A. Willam, C. Egger-Danner, and A. C. Sørensen. 2016. Reproductive technologies combine well with genomic selection in dairy breeding programs. J. Dairy Sci. 99:1331-1340. https: //doi.org/10.3168/jds.2015-9437.

Veroneze, R., J. Bastiaansen, E. F. Knol, S. E. F. Guimarães, F. F. Silva, B. Harlizius, M. S. Lopes, and P. S. Lopes. 2014. Linkage disequilibrium patterns and persistence of phase in purebred and crossbred pig (Sus scrofa) populations. BMC Genet. 15:126. https: //doi.org/10.1186/s12863-014-0126-3.

Villa-Angulo, R., L. K. Matukumalli, C. A. Gill, J. Choi, C. P. Van Tassell, and J. J. Grefenstette. 2009. High-resolution haplotype block structure in the cattle genome. BMC Genet. 10:19. https:// doi.org/10.1186/1471-2156-10-19.

\section{ORCIDS}

J. R. Thomasen (1) https://orcid.org/0000-0001-7068-0572

H. Liu (1) https://orcid.org/0000-0002-0761-326X 